\title{
Path Integral Approach to Noncommutative Quantum Mechanics
}

\author{
Branko Dragovich \\ Institute of Physics, P.O.Box 57, 11001 Belgrade \\ Serbia and Montenegro \\ E-mail: dragovich@phy.bg.ac.yu \\ Zoran Rakić \\ Faculty of Mathematics, University of Belgrade \\ Studentski trg 16, P.O.Box 550, 11001 Belgrade \\ Serbia and Montenegro \\ E-mail: zrakic@matf.bg.ac.yu
}

\begin{abstract}
We consider Feynman's path integral approach to quantum mechanics with a noncommutativity in position and momentum sectors of the phase space. We show that a quantum-mechanical system with this kind of noncommutativity is equivalent to the another one with usual commutative coordinates and momenta. We found connection between quadratic classical Hamiltonians, as well as Lagrangians, in their commutative and noncommutative regimes. The general procedure to compute Feynman's path integral on this noncommutative phase space with quadratic Lagrangians (Hamiltonians) is presented. Using this approach, a particle in a constant field, ordinary and inverted harmonic oscillators are elaborated in detail.
\end{abstract}

\section{INTRODUCTION}

Quantum theories with noncommuting position and momentum coordinates have been investigated intensively during the recent years. Most of the re- 
search has been devoted to noncommutative (NC) field theory (for a review, see e.g. [1]). Noncommutative quantum mechanics (NCQM) has been also investigated, because it can be regarded as the corresponding one-particle nonrelativistic sector of $\mathrm{NC}$ quantum field theory and with relevance to concrete quantum systems.

In a very general NCQM one has that not only $\left[\hat{x_{a}}, \hat{p_{b}}\right] \neq 0$, but also $\left[\hat{x_{a}}, \hat{x_{b}}\right] \neq 0$ and $\left[\hat{p_{a}}, \hat{p_{b}}\right] \neq 0$. We consider here $D$-dimensional NCQM which is based on the following algebra:

$$
\left[\hat{x_{a}}, \hat{p_{b}}\right]=i \hbar\left(\delta_{a b}-\frac{1}{4} \theta_{a c} \sigma_{c b}\right), \quad\left[\hat{x_{a}}, \hat{x_{b}}\right]=i \hbar \theta_{a b}, \quad\left[\hat{p_{a}}, \hat{p_{b}}\right]=i \hbar \sigma_{a b}
$$

where $\Theta=\left(\theta_{a b}\right)$ and $\Sigma=\left(\sigma_{a b}\right)$ are the antisymmetric matrices with constant elements.

We investigate the Feynman path integral [2] approach to NCQM,

$$
\mathcal{K}\left(x^{\prime \prime}, t^{\prime \prime} ; x^{\prime}, t^{\prime}\right)=\int_{x^{\prime}}^{x^{\prime \prime}} \exp \left(\frac{i}{\hbar} \int_{t^{\prime}}^{t^{\prime \prime}} L(\dot{q}, q, t) d t\right) \mathcal{D} q
$$

where $\mathcal{K}\left(x^{\prime \prime}, t^{\prime \prime} ; x^{\prime}, t^{\prime}\right)$ is the kernel of the unitary evolution operator $U(t)$ and $x^{\prime \prime}=q\left(t^{\prime \prime}\right), x^{\prime}=q\left(t^{\prime}\right)$ are end points. In ordinary quantum mechanics (OQM) Feynman path integral for quadratic Lagrangians can be evaluated analytically and the exact form [3] is

$$
\mathcal{K}\left(x^{\prime \prime}, t^{\prime \prime} ; x^{\prime}, t^{\prime}\right)=\frac{1}{(i h)^{\frac{D}{2}}} \sqrt{\operatorname{det}\left(-\frac{\partial^{2} \bar{S}}{\partial x_{a}^{\prime \prime} \partial x_{b}^{\prime}}\right)} \exp \left(\frac{2 \pi i}{h} \bar{S}\left(x^{\prime \prime}, t^{\prime \prime} ; x^{\prime}, t^{\prime}\right)\right),
$$

where $\bar{S}\left(x^{\prime \prime}, t^{\prime \prime} ; x^{\prime}, t^{\prime}\right)$ is the action for the classical trajectory.

In this article we search the form of an effective quadratic Lagrangian which corresponds to a system with noncommutative phase space coordinates (1). This is necessary to know before to employ Feynman's path integral method in NCQM. To this end, let us note that algebra (1) of operators $\hat{x_{a}}, \hat{p_{b}}$ can be replaced by the equivalent one

$$
\left[\hat{q_{a}}, \hat{k_{b}}\right]=i \hbar \delta_{a b}, \quad\left[\hat{q_{a}}, \hat{q_{b}}\right]=0, \quad\left[\hat{k_{a}}, \hat{k_{b}}\right]=0,
$$

where linear transformations

$$
\hat{x_{a}}=\hat{q_{a}}-\frac{\theta_{a b} \hat{k_{b}}}{2}, \quad \hat{p_{a}}=\hat{k_{a}}+\frac{\sigma_{a b} \hat{q_{b}}}{2}
$$


are used and summation over repeated indices is assumed. According to (11), (41) and (5), NCQM related to the quantum phase space $(\hat{p}, \hat{x})$ can be regarded as an OQM on the standard phase space $(\hat{k}, \hat{q})$. Similar aspects of noncommutativity with $\sigma_{a b}=0$ are considered in [4] and [5] .

\section{DYNAMICS ON NONCOMMUTATIVE PHASE SPACE AND PATH INTEGRAL}

Let the most general quadratic Lagrangian for a $D$-dimensional system be

$$
L(\dot{x}, x, t)=\frac{1}{2}\left(\dot{x}^{T} \alpha \dot{x}+\dot{x}^{T} \beta x+x^{T} \beta^{T} \dot{x}+x^{T} \gamma x\right)+\delta^{T} \dot{x}+\eta^{T} x+\phi,
$$

where coefficients of the $D \times D$ matrices $\alpha=\left(\left(1+\delta_{a b}\right) \alpha_{a b}(t)\right), \beta=\left(\beta_{a b}(t)\right), \gamma=$ $\left(\left(1+\delta_{a b}\right) \gamma_{a b}(t)\right), D$-dimensional vectors $\delta=\left(\delta_{a}(t)\right), \quad \eta=\left(\eta_{a}(t)\right)$ and a scalar $\phi=\phi(t)$ are some analytic functions of the time $t$. Matrices $\alpha$ and $\gamma$ are symmetric, $\alpha$ is nonsingular $(\operatorname{det} \alpha \neq 0)$ and index ${ }^{T}$ denotes transpose map.

The Lagrangian (6) can be presented in the more compact form:

$$
L(X, t)=\frac{1}{2} X^{T} M X+N^{T} X+\phi
$$

where $2 D \times 2 D$ matrix $M$ and $2 D$-dimensional vectors $X, N$ are defined as

$$
M=\left(\begin{array}{cc}
\alpha & \beta \\
\beta^{T} & \gamma
\end{array}\right), \quad X^{T}=\left(\dot{x}^{T}, x^{T}\right), \quad N^{T}=\left(\delta^{T}, \eta^{T}\right) .
$$

Using the equations $p_{a}=\frac{\partial L}{\partial \dot{x}_{a}}$, one can express $\dot{x}$ as $\dot{x}=\alpha^{-1}(p-\beta x-\delta)$. Since $\dot{x}$ is linear in $p$ and $x$, the corresponding classical Hamiltonian $H(p, x, t)=$ $p^{T} \dot{x}-L(\dot{x}, x, t)$ becomes also quadratic, i.e.

$$
H(p, x, t)=\frac{1}{2}\left(p^{T} A p+p^{T} B x+x^{T} B^{T} p+x^{T} C x\right)+D^{T} p+E^{T} x+F,
$$

where:

$$
\begin{array}{lll}
A=\alpha^{-1}, & B=-\alpha^{-1} \beta, & C=\beta^{T} \alpha^{-1} \beta-\gamma, \\
D=-\alpha^{-1} \delta, & E=\beta^{T} \alpha^{-1} \delta-\eta, & F=\frac{1}{2} \delta^{T} \alpha^{-1} \delta-\phi .
\end{array}
$$

Due to the symmetry of matrices $\alpha$ and $\gamma$ one has that matrices $A=$ $\left(\left(1+\delta_{a b}\right) A_{a b}(t)\right)$ and $C=\left(\left(1+\delta_{a b}\right) C_{a b}(t)\right)$ are also symmetric $\left(A^{T}=A, C^{T}=\right.$ 
$C$ ). The nonsingular (det $\alpha \neq 0$ ) Lagrangian $L(\dot{x}, x, t)$ implies nonsingular ( $\operatorname{det} A \neq 0)$ Hamiltonian $H(p, x, t)$. Note that the inverse map, i.e. $H \rightarrow L$, is given by the same relations (10).

The Hamiltonian (9) can be also presented in the compact form

$$
H(\Pi, t)=\frac{1}{2} \Pi^{T} \mathcal{M} \Pi+\mathcal{N}^{T} \Pi+F,
$$

where matrix $\mathcal{M}$ and vectors $\Pi, \mathcal{N}$ are

$$
\mathcal{M}=\left(\begin{array}{cc}
A & B \\
B^{T} & C
\end{array}\right), \quad \Pi^{T}=\left(p^{T}, x^{T}\right), \quad \mathcal{N}^{T}=\left(D^{T}, E^{T}\right) .
$$

One can easily show that

$$
\mathcal{M}=\sum_{i=1}^{3} \Upsilon_{i}^{T}(M) M \Upsilon_{i}(M)
$$

where

$\Upsilon_{1}(M)=\left(\begin{array}{rr}\alpha^{-1} & 0 \\ 0 & -I\end{array}\right), \Upsilon_{2}(M)=\left(\begin{array}{rr}0 & \alpha^{-1} \beta \\ 0 & 0\end{array}\right), \Upsilon_{3}(M)=\left(\begin{array}{rr}0 & 0 \\ 0 & i \sqrt{2} I\end{array}\right)$.

One has also $\mathcal{N}=Y(M) N$, where

$$
Y(M)=\left(\begin{array}{rr}
-\alpha^{-1} & 0 \\
\beta^{T} \alpha^{-1} & -I
\end{array}\right)=-\Upsilon_{1}(M)+\Upsilon_{2}^{T}(M)+i \sqrt{2} \Upsilon_{3}(M)
$$

and $F=N^{T} Z(M) N-\phi$, where

$$
Z(M)=\left(\begin{array}{cc}
\frac{1}{2} \alpha^{-1} & 0 \\
0 & 0
\end{array}\right)=\frac{1}{2} \Upsilon_{1}(M)-\frac{i}{2 \sqrt{2}} \Upsilon_{3}(M) .
$$

Eqs. (5) can be rewritten in the compact form as

$$
\hat{\Pi}=\Xi \hat{K}, \quad \Xi=\left(\begin{array}{cc}
I & \frac{1}{2} \Sigma \\
-\frac{1}{2} \Theta & I
\end{array}\right), \quad \hat{K}=\left(\begin{array}{c}
\hat{k} \\
\hat{q}
\end{array}\right) .
$$

Since Hamiltonians depend on canonical variables, the transformation (16) lead to the transformation of Hamiltonians (92) and (11). To this end, let us quantize the Hamiltonian (92) and it easily becomes $H(\hat{p}, \hat{x}, t)=\frac{1}{2}\left(\hat{p}^{T} A \hat{p}\right.$ 
$\left.+\hat{p}^{T} B \hat{x}+\hat{x}^{T} B^{T} \hat{p}+\hat{x}^{T} C \hat{x}\right)+D^{T} \hat{p}+E^{T} \hat{x}+F$ because (9) is already written in the Weyl symmetric form.

Performing linear transformations (5) in the above Hamiltonian we again obtain quadratic quantum Hamiltonian

$$
\begin{aligned}
H_{\theta \sigma}(\hat{k}, \hat{q}, t)= & \frac{1}{2}\left(\hat{k}^{T} A_{\theta \sigma} \hat{k}+\hat{k}^{T} B_{\theta \sigma} \hat{q}+\hat{q}^{T} B_{\theta \sigma}^{T} \hat{k}+\hat{q}^{T} C_{\theta \sigma} \hat{q}\right) \\
& +D_{\theta \sigma}^{T} \hat{k}+E_{\theta \sigma}^{T} \hat{q}+F_{\theta \sigma},
\end{aligned}
$$

where

$$
\begin{array}{ll}
A_{\theta \sigma}=A-\frac{1}{2} B \Theta+\frac{1}{2} \Theta B^{T}-\frac{1}{4} \Theta C \Theta, & D_{\theta \sigma}=D+\frac{1}{2} \Theta E \\
B_{\theta \sigma}=B+\frac{1}{2} \Theta C+\frac{1}{2} A \Sigma+\frac{1}{4} \Theta B^{T} \Sigma, & E_{\theta \sigma}=E-\frac{1}{2} \Sigma D \\
C_{\theta \sigma}=C-\frac{1}{2} \Sigma B+\frac{1}{2} B^{T} \Sigma-\frac{1}{4} \Sigma A \Sigma, & F_{\theta \sigma}=F .
\end{array}
$$

Note that for the nonsingular Hamiltonian $H(\hat{p}, \hat{x}, t)$ and for sufficiently small $\theta_{a b}$ the Hamiltonian $H_{\theta \sigma}(\hat{k}, \hat{q}, t)$ is also nonsingular. Classical analogue of (17) maintains the same form $H_{\theta \sigma}(k, q, t)=\frac{1}{2}\left(k^{T} A_{\theta \sigma} k+k^{T} B_{\theta \sigma} q+q^{T} B_{\theta \sigma}^{T} k\right.$ $\left.+q^{T} C_{\theta \sigma} q\right)+D_{\theta \sigma}^{T} k+E_{\theta \sigma}^{T} q+F_{\theta \sigma}$.

In the more compact form, Hamiltonian (17) is

$$
\hat{H}_{\theta \sigma}(\hat{K}, t)=\frac{1}{2} \hat{K}^{T} \mathcal{M}_{\theta \sigma} \hat{K}+\mathcal{N}_{\theta \sigma}^{T} \hat{K}+F_{\theta \sigma}
$$

where $2 D \times 2 D$ matrix $\mathcal{M}_{\theta \sigma}$ and $2 D$-dimensional vectors $\hat{K}, \mathcal{N}_{\theta \sigma}$ are

$$
\mathcal{M}_{\theta \sigma}=\left(\begin{array}{cc}
A_{\theta \sigma} & B_{\theta \sigma} \\
B_{\theta \sigma}^{T} & C_{\theta \sigma}
\end{array}\right), \quad \hat{K}^{T}=\left(\hat{k}^{T}, \hat{q}^{T}\right), \quad \mathcal{N}_{\theta \sigma}^{T}=\left(D_{\theta \sigma}^{T}, E_{\theta \sigma}^{T}\right) .
$$

From (11), (16) and (19) one can find connections between $\mathcal{M}_{\theta \sigma}, \mathcal{N}_{\theta \sigma}, F_{\theta \sigma}$ and $\mathcal{M}, \mathcal{N}, F$, which are given by the following relations:

$$
\mathcal{M}_{\theta \sigma}=\Xi^{T} \mathcal{M} \Xi, \quad \mathcal{N}_{\theta \sigma}=\Xi^{T} \mathcal{N}, \quad F_{\theta \sigma}=F
$$

To compute a path integral one can start from its Hamiltonian formulation on the phase space. However, such path integral on a phase space 
can be reduced to the Lagrangian path integral on configuration space whenever Hamiltonian is a quadratic polynomial with respect to momentum $p$ (see, e.g. [5]). Hence, we need the corresponding classical Lagrangians related to the Hamiltonians (17) and (19). Using equations $\dot{q}_{a}=\frac{\partial H_{\theta \sigma}}{\partial p_{a}}$ which give $k=A_{\theta \sigma}^{-1}\left(\dot{q}-B_{\theta \sigma} q-D_{\theta \sigma}\right)$ we can pass from Hamiltonian (17) to the corresponding Lagrangian by relation $L_{\theta \sigma}(\dot{q}, q, t)=k^{T} \dot{q}-H_{\theta \sigma}(k, q, t)$. Note that coordinates $q_{a}$ and $x_{a}$ coincide when $\theta=\sigma=0$. Performing necessary computations we obtain

$$
\begin{aligned}
L_{\theta \sigma}(\dot{q}, q, t)= & \frac{1}{2}\left(\dot{q}^{T} \alpha_{\theta \sigma} \dot{q}+\dot{q}^{T} \beta_{\theta \sigma} q+q^{T} \beta_{\theta \sigma}^{T} \dot{q}+q^{T} \gamma_{\theta \sigma} q\right) \\
& +\delta_{\theta \sigma}^{T} \dot{q}+\eta_{\theta \sigma}^{T} q+\phi_{\theta \sigma},
\end{aligned}
$$

or in the compact form:

$$
L_{\theta \sigma}(Q, t)=\frac{1}{2} Q^{T} M_{\theta \sigma} Q+N_{\theta \sigma}^{T} Q+\phi_{\theta \sigma},
$$

where

$$
M_{\theta \sigma}=\left(\begin{array}{cc}
\alpha_{\theta \sigma} & \beta_{\theta \sigma} \\
\beta_{\theta \sigma}^{T} & \gamma_{\theta \sigma}
\end{array}\right), \quad Q^{T}=\left(\dot{q}^{T}, q^{T}\right), \quad N^{T}=\left(\delta_{\theta \sigma}^{T}, \eta_{\theta \sigma}^{T}\right)
$$

Then the connection between $M_{\theta \sigma}, N_{\theta \sigma}, \phi_{\theta \sigma}$ and $M, N, \phi$ are given by the following relations:

$$
M_{\theta \sigma}=\sum_{i, j=1}^{3} \Xi_{i j}^{T} M \Xi_{i j}, \quad \Xi_{i j}=\Upsilon_{i}(M) \Xi \Upsilon_{j}\left(\mathcal{M}_{\theta \sigma}\right),
$$

and

$$
N_{\theta \sigma}=Y\left(\mathcal{M}_{\theta \sigma}\right) \Xi^{T} Y(M) N, \quad \phi_{\theta \sigma}=\mathcal{N}_{\theta \sigma}^{T} Z\left(\mathcal{M}_{\theta \sigma}\right) \mathcal{N}_{\theta \sigma}-F .
$$

In more detail, the connection between coefficients of the Lagrangians $L_{\theta \sigma}$ and $L$ is given by the relations:

$$
\begin{aligned}
\alpha_{\theta \sigma}= & {\left[\alpha^{-1}-\frac{1}{2}\left(\Theta \beta^{T} \alpha^{-1}-\alpha^{-1} \beta \Theta\right)-\frac{1}{4} \Theta\left(\beta^{T} \alpha^{-1} \beta-\gamma\right) \Theta\right]^{-1}, } \\
\beta_{\theta \sigma}= & \alpha_{\theta \sigma}\left(\alpha^{-1} \beta-\frac{1}{2}\left(\alpha^{-1} \Sigma-\Theta \gamma+\Theta \beta^{T} \alpha^{-1} \beta\right)+\frac{1}{4} \Theta \beta^{T} \alpha^{-1} \Sigma\right), \\
\gamma_{\theta \sigma}= & \gamma+\beta_{\theta \sigma}^{T} \alpha_{\theta \sigma}^{-1} \beta_{\theta \sigma}-\beta^{T} \alpha^{-1} \beta+\frac{1}{4} \Sigma \alpha^{-1} \Sigma \\
& -\frac{1}{2}\left(\Sigma \alpha^{-1} \beta-\beta^{T} \alpha^{-1} \Sigma\right),
\end{aligned}
$$




$$
\begin{aligned}
\delta_{\theta \sigma} & =\alpha_{\theta \sigma}\left(\alpha^{-1} \delta+\frac{1}{2}\left(\Theta \eta-\Theta \beta^{T} \alpha^{-1} \delta\right)\right), \\
\eta_{\theta \sigma} & =\eta+\beta_{\theta \sigma}^{T} \alpha_{\theta \sigma}^{-1} \delta_{\theta \sigma}-\beta^{T} \alpha^{-1} \delta-\frac{1}{2} \Sigma \alpha^{-1} \delta \\
\phi_{\theta \sigma} & =\phi+\frac{1}{2} \delta_{\theta \sigma}^{T} \alpha_{\theta \sigma}^{-1} \delta_{\theta \sigma}-\frac{1}{2} \delta^{T} \alpha^{-1} \delta .
\end{aligned}
$$

Note that $\alpha_{\theta \sigma}, \delta_{\theta \sigma}$ and $\phi_{\theta \sigma}$ do not depend on $\sigma$.

If we know Lagrangian (6) and algebra (11) we can obtain the corresponding effective Lagrangian (22) suitable for the path integral in NCQM. Exploiting the Euler-Lagrange equations $\frac{\partial L_{\theta \sigma}}{\partial q_{a}}-\frac{d}{d t} \frac{\partial L_{\theta \sigma}}{\partial \dot{q}_{a}}=0$ one can obtain classical trajectory $q_{a}=q_{a}(t)$ connecting end points $x^{\prime}=q\left(t^{\prime}\right)$ and $x^{\prime \prime}=q\left(t^{\prime \prime}\right)$, and the corresponding action $\bar{S}_{\theta \sigma}\left(x^{\prime \prime}, t^{\prime \prime} ; x^{\prime}, t^{\prime}\right)=\int_{t^{\prime}}^{t^{\prime \prime}} L_{\theta \sigma}(\dot{q}, q, t) d t$. Path integral in NCQM is a direct analogue of (3) and its exact expression in the form of quadratic actions $\bar{S}_{\theta \sigma}\left(x^{\prime \prime}, t^{\prime \prime} ; x^{\prime}, t^{\prime}\right)$ is

$$
\mathcal{K}_{\theta \sigma}\left(x^{\prime \prime}, t^{\prime \prime} ; x^{\prime}, t^{\prime}\right)=\frac{1}{(i h)^{\frac{D}{2}}} \sqrt{\operatorname{det}\left(-\frac{\partial^{2} \bar{S}_{\theta \sigma}}{\partial x_{a}^{\prime \prime} \partial x_{b}^{\prime}}\right)} \exp \left(\frac{2 \pi i}{h} \bar{S}_{\theta \sigma}\left(x^{\prime \prime}, t^{\prime \prime} ; x^{\prime}, t^{\prime}\right)\right)
$$

\subsection{A particle in a constant field and on noncommuta- tive phase space}

The starting Lagrangian is

$$
L(\dot{x}, x)=\frac{m}{2}\left(\dot{x}_{1}^{2}+\dot{x}_{2}^{2}\right)-\eta_{1} x_{1}-\eta_{2} x_{2} .
$$

Using (27), one can easily find the Lagrangian $L_{\theta \sigma}(\dot{q}, q, t)$ :

$$
\begin{aligned}
L_{\theta \sigma}= & \frac{m}{2}\left(\dot{q}_{1}^{2}+\dot{q}_{2}^{2}\right)+\frac{\sigma}{2}\left(q_{1} \dot{q}_{2}-q_{2} \dot{q}_{1}\right)+\frac{m \theta}{2}\left(\eta_{1} \dot{q}_{2}+\eta_{2} \dot{q}_{1}\right) \\
& -\left(1-\frac{\theta \sigma}{4}\right)\left(\eta_{1} q_{1}+\eta_{2} q_{2}\right)+\frac{m \theta^{2}}{8}\left(\eta_{1}{ }^{2}+\eta_{2}{ }^{2}\right) .
\end{aligned}
$$

The corresponding equations of motion are $\ddot{q}_{1}-\xi \dot{q}_{2}+\eta_{1} \phi=0, \ddot{q}_{2}+\xi \dot{q}_{1}+$ $\eta_{2} \phi=0$, where $\xi=\frac{\sigma}{m}, \phi=\frac{1}{m}\left(1-\frac{\theta \sigma}{4}\right)$. One can transform the above system of equations to $q_{1}^{(3)}+\xi^{2} \dot{q}_{1}+\eta_{2} \xi \phi=0, \quad q_{2}^{(3)}+\xi^{2} \dot{q}_{2}-\eta_{1} \xi \phi=0$, Their solutions are: $q_{1}(t)=C_{1}+C_{2} \cos [\xi t]+C_{3} \sin [\xi t]-\frac{\phi \eta_{2}}{\xi} t, \quad q_{2}(t)=$ $D_{1}+D_{2} \cos [\xi t]+D_{3} \sin [\xi t]+\frac{\phi \eta_{1}}{\xi} t$, where $C_{1}, C_{2}, C_{3}, D_{1}, D_{2}$ and $D_{3}$ are 
constants. Since the functions $q_{1}$ and $q_{2}$ have to satisfy coupled differential equations one has $D_{2}=C_{3}, \quad D_{3}=-C_{2}$. Employing the conditions $q_{1}(0)=$ $x_{1}^{\prime}, \quad q_{1}(T)=x_{1}^{\prime \prime}, \quad q_{2}(0)=x_{2}^{\prime}, \quad q_{2}(T)=x_{2}^{\prime \prime}$ one obtains:

$$
\begin{aligned}
& C_{1}=\frac{\left(x_{1}^{\prime}+x_{1}^{\prime \prime}\right) \xi+\phi \eta_{2} T}{2 \xi}-\frac{\left(\left(x_{2}^{\prime}-x_{2}^{\prime \prime}\right) \xi+\phi \eta_{1} T\right)}{2 \xi} \cot \left[\frac{\xi T}{2}\right], \\
& C_{3}=\frac{\left(x_{2}^{\prime}-x_{2}^{\prime \prime}\right) \xi+\phi \eta_{1} T}{2 \xi}-\frac{\left(\left(x_{1}^{\prime}-x_{1}^{\prime \prime}\right) \xi-\phi \eta_{2} T\right)}{2 \xi} \cot \left[\frac{\xi T}{2}\right], \\
& D_{1}=\frac{\left(x_{2}^{\prime}+x_{2}^{\prime \prime}\right) \xi-\phi \eta_{1} T}{2 \xi}-\frac{\left(\left(x_{1}^{\prime \prime}-x_{1}^{\prime}\right) \xi+\phi \eta_{2} T\right)}{2 \xi} \cot \left[\frac{\xi T}{2}\right], \\
& D_{3}=\frac{\left(x_{1}^{\prime \prime}-x_{1}^{\prime}\right) \xi+\phi \eta_{2} T}{2 \xi}-\frac{\left(\left(x_{2}^{\prime}-x_{2}^{\prime \prime}\right) \xi+\phi \eta_{1} T\right)}{2 \xi} \cot \left[\frac{\xi T}{2}\right] .
\end{aligned}
$$

The Lagrangian for classical trajectory is $L_{\theta \sigma}(\dot{q}, q)=-\frac{m \xi \phi t}{2}\left(\left(C_{3} \eta_{1}+\right.\right.$ $\left.\left.D_{3} \eta_{2}\right) \cos [\xi t]+\left(D_{3} \eta_{1}-C_{3} \eta_{2}\right) \sin [\xi t]\right)-\frac{m}{2}\left(\left(C_{3} D_{1}-C_{1} D_{3}\right) \xi^{2}+(\theta \xi+\right.$ $\left.3 \phi)\left(C_{3} \eta_{2}-D_{3} \eta_{1}\right) \cos [\xi t]+\left(C_{1} C_{3}+D_{1} D_{3}\right) \xi^{2}+(\theta \xi+3 \phi)\left(C_{3} \eta_{1}+D_{3} \eta_{2}\right) \sin [\xi t]\right)-$ $\frac{m \phi}{2}\left(C_{1} \eta_{1}+D_{1} \eta_{2}\right)+\frac{m(\theta \xi+2 \phi)^{2}\left(\eta_{1}^{2}+\eta_{2}^{2}\right)}{8 \xi^{2}}$. Using this Lagrangian we compute the classical action

$$
\begin{aligned}
& \bar{S}_{\theta \sigma}\left(x^{\prime \prime}, T ; x^{\prime}, 0\right)=\int_{0}^{T} L_{\theta \sigma}(\dot{q}, q) d t=\frac{m \xi}{2}\left(x_{2}^{\prime \prime} x_{1}^{\prime}-x_{1}^{\prime \prime} x_{2}^{\prime}\right)+\frac{m \xi \cot \left[\frac{\xi T}{2}\right]}{4} \\
& \times\left(\left(x_{1}^{\prime \prime}-x_{1}^{\prime}\right)^{2}+\left(x_{2}^{\prime \prime}-x_{2}^{\prime}\right)^{2}\right)+\frac{2 \lambda+m \theta \xi-\lambda \xi T \cot \left[\frac{\xi T}{2}\right]}{2 \xi} \\
& \times\left(\left(x_{2}^{\prime \prime}-x_{2}^{\prime}\right) \eta_{1}-\left(x_{1}^{\prime \prime}-x_{1}^{\prime}\right) \eta_{2}\right)-\frac{\lambda T}{2}\left(\left(x_{1}^{\prime \prime}+x_{1}^{\prime}\right) \eta_{1}+\left(x_{2}^{\prime \prime}+x_{2}^{\prime}\right) \eta_{2}\right) \\
& +\frac{T}{8 m \xi^{2}}\left(2 \xi \lambda^{2} T \cot \left[\frac{\xi T}{2}\right]+\left(m^{2} \theta^{2} \xi^{2}-4 \lambda^{2}\right)\right)\left(\eta_{1}^{2}+\eta_{2}^{2}\right) .
\end{aligned}
$$

Finally, we have

$$
\mathcal{K}_{\theta \sigma}\left(x^{\prime \prime}, T ; x^{\prime}, 0\right)=\frac{m|\xi|}{2 i h\left|\sin \left[\frac{\xi T}{2}\right]\right|} \exp \left(\frac{2 \pi i}{h} \bar{S}_{\theta \sigma}\left(x^{\prime \prime}, T ; x^{\prime}, 0\right)\right) .
$$

\subsection{Ordinary and inverted harmonic oscillator on non- commutative plane}

The Lagrangian in the question is

$$
L(\dot{x}, x)=\frac{m}{2}\left(\dot{x}_{1}^{2}+\dot{x}_{2}^{2}\right)-\varepsilon \frac{m \omega^{2}}{2}\left(x_{1}^{2}+x_{2}^{2}\right),
$$


where $\varepsilon=1$ and $\varepsilon=-1$ are for ordinary and inverted harmonic oscillators, respectively. Using formulas (27), one can easily find the corresponding noncommutative Lagrangian

$$
\begin{aligned}
L_{\theta \sigma}(\dot{q}, q)= & \frac{m}{2 \kappa}\left(\dot{q}_{1}^{2}+\dot{q}_{2}^{2}\right)+\frac{\sigma+\varepsilon m^{2} \omega^{2} \theta}{2 \kappa}\left(\dot{q}_{2} q_{1}-\dot{q}_{1} q_{2}\right) \\
& -\frac{\varepsilon m \omega^{2}}{2 \kappa} \lambda^{2}\left(q_{1}{ }^{2}+q_{2}{ }^{2}\right)
\end{aligned}
$$

where $\kappa=1+\frac{\varepsilon m^{2} \omega^{2} \theta^{2}}{4}$ and $\lambda=1-\frac{\theta \sigma}{4}$.

From (34), we obtain the Euler-Lagrange equations, $\ddot{q}_{1}-\chi \dot{q}_{2}+\mu q_{1}=$ $0, \quad \ddot{q}_{2}+\chi \dot{q}_{1}+\mu q_{2}=0$, where $\chi=\frac{\sigma}{m}+\varepsilon m \omega^{2} \theta, \quad \mu=\varepsilon \omega^{2} \lambda^{2}$. Let us note that these equations form a coupled system of second order differential equations, which is more complicated than in the commutative case $(\theta=\sigma=0)$. One can transform this system to $q_{1}^{(4)}+\left(\chi^{2}+2 \mu\right) q_{1}^{(2)}+\mu^{2} q_{1}=0, \quad q_{2}^{(4)}+\left(\chi^{2}+\right.$ $2 \mu) q_{2}^{(2)}+\mu^{2} q_{2}=0$. The solution of these equations for $|\theta \sigma|<4, \omega>0, \chi \neq 0$ and $\varepsilon>0$ has the form $\left(\nu=\frac{\chi^{2}+4 \mu}{4}\right)$ :

$$
\begin{aligned}
& q_{1}(t)=C_{1} \cos \left[\frac{\chi}{2} t\right] \cos [\sqrt{\varepsilon|\nu|} t]+C_{2} \cos \left[\frac{\chi}{2} t\right]|\sqrt{\varepsilon} \sin [\sqrt{\varepsilon|\nu|} t]| \\
& +C_{3} \sin \left[\frac{\chi}{2} t\right] \cos [\sqrt{\varepsilon|\nu|} t]+C_{4} \sin \left[\frac{\chi}{2} t\right]|\sqrt{\varepsilon} \sin [\sqrt{\varepsilon|\nu|} t]| \\
& q_{2}(t)=D_{1} \cos \left[\frac{\chi}{2} t\right] \cos [\sqrt{\varepsilon|\nu|} t]+D_{2} \cos \left[\frac{\chi}{2} t\right]|\sqrt{\varepsilon} \sin [\sqrt{\varepsilon|\nu|} t]| \\
& +D_{3} \sin \left[\frac{\chi}{2} t\right] \cos [\sqrt{\varepsilon|\nu|} t]+D_{4} \sin \left[\frac{\chi}{2} t\right]|\sqrt{\varepsilon} \sin [\sqrt{\varepsilon|\nu|} t]| .
\end{aligned}
$$

Let us note that in the case of inverted harmonic oscillator $(\varepsilon=-1)$ the trigonometric functions in $\sqrt{\varepsilon|\nu|} t$ from (35) become the corresponding hyperbolic functions in $\sqrt{|\nu|} t$. After imposing connections between $q_{1}$ and $q_{2}$ given by coupled differential equations, we obtain the following relations between constants $C$ and $D: D_{1}=C_{2}, \quad D_{2}=-C_{1}, \quad D_{3}=C_{4}, \quad D_{4}=-C_{3}$. The unknown constants $C_{1}, C_{2}, C_{3}$ and $C_{4}$ one can find from end conditions $q_{1}(0)=x_{1}^{\prime}, \quad q_{1}(T)=x_{1}^{\prime \prime}, \quad q_{2}(0)=x_{2}^{\prime}, \quad q_{2}(T)=x_{2}^{\prime \prime}$. Then one obtains the solutions

$$
\begin{aligned}
q_{1}= & \frac{1}{\sin [\sqrt{\varepsilon|\nu|} T]}\left(\left(x_{1}^{\prime \prime} \cos \left[\frac{\chi}{2}(t-T)\right]+x_{2}^{\prime \prime} \sin \left[\frac{\chi}{2}(t-T)\right]\right) \sin [\sqrt{\varepsilon|\nu|} t]\right. \\
& \left.-\left(x_{1}^{\prime} \cos \left[\frac{\chi t}{2}\right]+x_{2}^{\prime} \sin \left[\frac{\chi t}{2}\right]\right) \sin [\sqrt{\varepsilon|\nu|}(t-T)]\right)
\end{aligned}
$$




$$
\begin{aligned}
q_{2}= & \frac{1}{\sin [\sqrt{\varepsilon|\nu|} T]}\left(\left(x_{2}^{\prime \prime} \cos \left[\frac{\chi}{2}(t-T)\right]-x_{1}^{\prime \prime} \sin \left[\frac{\chi}{2}(t-T)\right]\right) \sin [\sqrt{\varepsilon|\nu|} t]\right. \\
& \left.+\left(-x_{2}^{\prime} \cos \left[\frac{\chi t}{2}\right]+x_{1}^{\prime} \sin \left[\frac{\chi t}{2}\right]\right) \sin [\sqrt{\varepsilon|\nu|}(t-T)]\right) .
\end{aligned}
$$

Inserting the above expressions and their time derivatives into (34) we find

$$
\begin{aligned}
& L_{\theta \sigma}(\dot{q}, q)=\frac{m \varepsilon \nu}{2 \kappa \sin ^{2}[\sqrt{\varepsilon|\nu|} T]}\left(\left(x_{1}^{\prime \prime 2}+x_{2}^{\prime \prime 2}\right) \cos [2 \sqrt{\varepsilon|\nu|} t]+\left(x_{1}^{\prime 2}+x_{2}^{\prime 2}\right)\right. \\
& \times \cos [2 \sqrt{\varepsilon|\nu|}(t-T)]-2 \cos [\sqrt{\varepsilon|\nu|}(2 t-T)]\left(\left(x_{1}^{\prime} x_{1}^{\prime \prime}+x_{2}^{\prime} x_{2}^{\prime \prime}\right)\right. \\
& \left.\left.\times \cos \left[\frac{\chi T}{2}\right]+\left(x_{1}^{\prime \prime} x_{2}^{\prime}-x_{1}^{\prime} x_{2}^{\prime \prime}\right) \sin \left[\frac{\chi T}{2}\right]\right)\right) .
\end{aligned}
$$

Using (38), we finally compute the corresponding classical action

$$
\begin{aligned}
& \bar{S}_{\theta \sigma}\left(x^{\prime \prime}, T ; x^{\prime}, 0\right)=\int_{0}^{T} L_{\theta \sigma}(\dot{q}, q) d t \\
= & \frac{m \sqrt{\varepsilon|\nu|}}{2 \kappa \sin [\sqrt{\varepsilon|\nu|} T]}\left(-2\left(\left(x_{1}^{\prime} x_{1}^{\prime \prime}+x_{2}^{\prime} x_{2}^{\prime \prime}\right) \cos \left[\frac{\chi T}{2}\right]\right.\right. \\
+ & \left.\left(x_{1}^{\prime 2}+x_{1}^{\prime \prime 2}+x_{2}^{\prime 2}+x_{2}^{\prime \prime 2}\right) \cos [\sqrt{\varepsilon|\nu|} T]+2\left(-x_{1}^{\prime \prime} x_{2}^{\prime}+x_{1}^{\prime} x_{2}^{\prime \prime}\right) \sin \left[\frac{\chi T}{2}\right]\right) .
\end{aligned}
$$

If we take into account expression (39) then we finally have

$$
\mathcal{K}_{\theta \sigma}\left(x^{\prime \prime}, T ; x^{\prime}, 0\right)=\frac{1}{i h} \frac{m|\sqrt{\varepsilon|\nu|}|}{\kappa|\sin [\sqrt{\varepsilon|\nu|} T]|} \exp \left(\frac{2 \pi i}{h} \bar{S}_{\theta \sigma}\left(x^{\prime \prime}, T ; x^{\prime}, 0\right)\right) \text {. }
$$

\section{CONCLUDING REMARKS}

Let us mention that taking $\sigma=0, \theta=0$ in the above formulas we recover expressions for the Lagrangian $L(\dot{q}, q)$, action $\bar{S}\left(x^{\prime \prime}, T ; x^{\prime}, 0\right)$ and probability amplitude $\mathcal{K}\left(x^{\prime \prime}, T ; x^{\prime}, 0\right)$ of the ordinary commutative case.

Note that a similar path integral approach with $\sigma=0$ has been considered in the context of the Aharonov-Bohm effect, the Casimir effect, a quantum system in a rotating frame, and the Hall effect (for references on these and some other related subjects, see [4, 5]). Our investigation includes all systems with quadratic Lagrangians (Hamiltonians). 
On the basis of the expressions presented in this article, there are many possibilities to discuss noncommutative quantum-mechanical systems with respect to various values of noncommutativity parameters $\theta$ and $\sigma$. For example, in the case $\theta \sigma=4$ one has a critical point in the above our particular twodimensional models (see also [6]).

\section{Acknowledgments}

B.D. would like to thank the organizers of the Fifth International Workshop "Lie Theory and its Applications in Physics" for invitation and hospitality, as well as for a stimulating and pleasant atmosphere. A part of this paper was done during stay of B.D. in the Steklov Mathematical Institute, Moscow. The work on this article was partially supported by the Serbian Ministry of Science, Technologies and Development under contracts No 1426 and No 1646. The work of B.D. was also supported in part by RFFI grant 02-01-01084.

\section{References}

[1] M. R. Douglas and N. A. Nekrasov, Rev. Mod. Phys. 73, 977 (2001) ; hep-th/0106048.

[2] R. P. Feynman and A. R. Hibbs, Quantum Mechanics and Path Integrals, (McGraw-Hill Book Company, New York, 1965).

[3] C. Grosche and F. Steiner, Handbook of Feynman Path Integrals (SpringerVerlag, Berlin, 1998).

[4] B. Dragovich and Z. Rakić, Lagrangian Aspects of Quantum Dynamics on a Noncommutative Space; hep-th/0302167.

[5] B. Dragovich and Z. Rakić , Path Integrals in Noncommutative Quantum Mechanics, to appear in the Theoretical and Mathematical Physics; hep-th/0309204.

[6] S. Bellucci, A. Nersessian and C. Sochichiu, Phys.Lett. B 522345 (2001); hep-th/0106138. 